\title{
Magnetocaloric effect and its implementation in critical behaviour study of $\mathrm{La}_{0.67} \mathrm{Ca}_{0.33} \mathrm{Mn}_{0.9} \mathrm{Fe}_{0.1} \mathrm{O}_{3}$
}

\author{
R M'NASSRI ${ }^{1,2}$ (D) \\ ${ }^{1}$ Higher Institute of Applied Sciences and Technology of Kasserine, Kairouan University, BP 471, 1200 Kasserine, Tunisia \\ ${ }^{2}$ Laboratoire de Physico-Chimie des Matériaux, Département de Physique, Faculté des Sciences de Monastir, \\ Université de Monastir, 5019 Monastir, Tunisia
}

MS received 4 August 2015; accepted 8 February 2016

\begin{abstract}
The magnetocaloric effect (MCE) and the field dependence of the magnetic entropy changes in the perovskite-type $\mathrm{La}_{0.67} \mathrm{Ca}_{0.33} \mathrm{Mn}_{0.9} \mathrm{Fe}_{0.1} \mathrm{O}_{3}$ were studied using the phenomenological model. The model parameters were determined from the magnetization data adjustment and used to give better fits to magnetic transition and to calculate the magnetocaloric properties. The entropy curves have been observed to behave a symmetrical broadning of $\Delta S_{\mathrm{M}}$ peak with the increase in magnetic field. The values of maximum magnetic entropy change, full-width at half-maximum, relative cooling power (RCP) and the refrigerant capacity $(\mathrm{RC})$, at several magnetic field variations, were calculated. The maximum magnetic entropy change of $1.17 \mathrm{~J} \mathrm{~kg}^{-1} \mathrm{~K}^{-1}$ was obtained for $3 \mathrm{~T}$. The theoretical calculations were compared with the available experimental data. The results were found to be in good accordance. The critical exponents associated with ferromagnetic transition have been determined from the MCE methods. By using the field dependence of $\Delta S_{\max } \approx a\left(\mu_{0} H\right)^{n}$ and the $\mathbf{R C P} \approx v\left(\mu_{0} H\right)^{w}$, the critical behaviour of $\mathrm{La}_{0.67} \mathrm{Ca}_{0.33} \mathrm{Mn}_{0.9} \mathrm{Fe}_{0.1} \mathrm{O}_{3}$ was investigated. From the analysis of the relationship between the local exponent $n$ and $w$, other exponents $\beta, \gamma$ and $\delta$ were calculated. Our results indicated that the ferromagnetic coupling in the $\mathrm{La}_{0.67} \mathrm{Ca}_{0.33} \mathrm{Mn}_{0.9} \mathrm{Fe}_{0.1} \mathrm{O}_{3}$ can be well described by the 3D Heisenberg model. This reflects an existence of ferromagnetic short-range order in the sample.
\end{abstract}

Keywords. Model; manganites; magnetization; magnetocaloric effect; critical exponent.

\section{Introduction}

Large number of magnetocaloric effect (MCE) materials have attracted much attention owing to their numerous potential advantages over vapour-compression refrigeration: higher chemical stability, easy fabrication, longer usage time, low noise, etc. [1-5]. For an overview on the experimental and the theoretical aspects of the (MCE), see references [6,7]. A lot of work has been carried out by various researchers with the aim to understand physics of perovskite-manganites and to explore possible applications such as MCE [8-12]. These materials exhibit a large magnetization change under the application of a magnetic field. They transform from a magnetically disordered state to an ordered state (at a temperature close to its critical temperature), and consequently large magnetic entropy changes occur [1]. Several compounds with a high MCE can be elaborated at different (low, intermediate and high) temperatures, and the major problem remains to get the samples with a high MCE at a low external magnetic field, which is advantageous for application as magnetic cooling, working in the fields produced by permanent magnets [13]. Manganites are promising candidate materials to satisfy this requirement and might be of the option of future magnetic cooling [14-16]. These perovskites

(rafik_mnassri@yahoo.fr) are characterized by the strong competition among a ferromagnetic double-exchange (DE) interaction, an antiferromagnetic super-exchange interaction and the spin-phonon coupling [17-19]. Magnetic and magnetocaloric properties of these interactions are determined by intrinsic parameters such as, doping level [20], average cationic size [21], cationic disorder [22], grain boundary [23], sintering temperature [24], particle size [25] and oxygen stoichiometry [26].

Phan et al [27] studied in their review the MCE in manganites systems and they have explained that the large MCE in the perovskite manganites can originate from the spinlattice coupling related to the magnetic ordering process. This strong coupling is evidenced by the lattice changes accompanying the magnetic transitions in these manganites; the lattice structural change in $\mathrm{Mn}-\mathrm{O}-\mathrm{Mn}$ bond angles and $\mathrm{Mn}-\mathrm{O}$ bond distances with temperature, which results in a variation of the volume, can cause an additional change in the magnetic properties of the material. There is no doubt that manganese ions play a crucial role in the DE process. Hence, it is worthwhile to investigate the influences of the replacement of Mn by other transition metals. Several works have been made on the effects of the substitution at Mn site by various elements. They suggested that the introduction of a transition element $\mathrm{Cr}[28,29]$, Co [30,31] and $\mathrm{Fe}[32,33]$ commonly affected the magnetic and electrical properties in manganites systems. It is found that when modifying the 
Mn element in ferromagnetic manganites always leads to a reduction in $T_{\mathrm{C}}$ and magnetization, an increase in the resistivity and ultimately insulating state at low temperatures for high substitutions.

To obtain a large $\Delta S_{\mathrm{M}}$ material without a thermal and magnetic hysteresis which is workable in a wide range of temperatures, Mukadam et al have studied the effect of the doping of $\mathrm{Fe}$ in $\mathrm{La}_{0.67} \mathrm{Ca}_{0.33} \mathrm{MnO}_{3}$ manganites [34]. Iron was chosen as the dopant because $\mathrm{Mn}^{3+}$ and $\mathrm{Fe}^{3+}$ have ionic radii that are close to each other and hence the crystal structure of the material remains almost unaltered [34]. Since the spin, charge, lattice and orbital degrees of freedom are intimately linked to the $\mathrm{Mn}$ ion, any perturbation at the Mn site is expected to modify ground state properties of such manganites. In a mixed system of $\mathrm{Fe}$ and $\mathrm{Mn}$, the $\mathrm{Fe}^{3+}$ ions do not participate in the DE interaction with $\mathrm{Mn}^{3+}$ ions. Thus, the ferromagnetic DE interaction is weakened to the profit of superexchange interactions which favours the antiferromagnetic coupling between $\mathrm{Mn}^{4+}-\mathrm{O}-\mathrm{Mn}^{4+}, \mathrm{Mn}^{4+}-\mathrm{O}-\mathrm{Fe}^{3+}$ and $\mathrm{Fe}^{3+}-\mathrm{O}-\mathrm{Fe}^{3+}$.

The knowledge of field dependence of magnetic entropy change $\Delta S_{\mathrm{M}}$ of a magnetic refrigerant material is important [35]. Understanding the field dependence can provide further clues to improve the performance of refrigerant materials in a lower field rather than those needed in already existing prototypes (generally 1-2 T). The field's dependence of the magnetic entropy change curve helps us to predict the response of a particular material under different extreme conditions, which can be useful for designing new materials for magnetic refrigeration [6]. Therefore, a study of the MCE for a particular material is not only important from a practical application point of view but also it provides a tool to understand the properties of the material. In particular, the details of the magnetic phase transition and critical behaviour can be obtained by studying the MCE of the material [36,37].

In this paper, the magnetocaloric properties and the critical behaviour for the optimized $\mathrm{La}_{0.67} \mathrm{Ca}_{0.33} \mathrm{Mn}_{0.9} \mathrm{Fe}_{0.1} \mathrm{O}_{3}$ compound at several magnetic fields was investigated. The polycrystalline sample was prepared by a solid-state reaction method [34].

\section{Model calculations}

According to the phenomenological model [38], the dependence of magnetization on variation of temperature and Curie temperature $T_{\mathrm{C}}$ is presented by

$$
\begin{aligned}
M\left(T, H=H_{\max }\right)= & \left(\frac{M_{\mathrm{i}}-M_{\mathrm{f}}}{2}\right)\left[\tanh \left(A\left(T_{\mathrm{C}}-T\right)\right)\right] \\
& +B T+C
\end{aligned}
$$

where $H_{\max }$ is the maximum external field, $T_{\mathrm{C}}$ the Curie temperature; $M_{\mathrm{i}} / M_{\mathrm{f}}$ the initial/final value of magnetization at ferromagnetic-paramagnetic transition as shown in figure 1. Here,

$$
A=2\left(\frac{B-S_{\mathrm{c}}}{M_{\mathrm{i}}-M_{\mathrm{f}}}\right),
$$

$B$ the magnetization sensitivity $\mathrm{d} M / \mathrm{d} T$ at ferromagnetic state before transition, $S_{\mathrm{c}}$ the magnetization sensitivity $\mathrm{d} M / \mathrm{d} T$ at Curie temperature $T_{\mathrm{C}}$, and $C=\left(\left(M_{\mathrm{i}}-M_{\mathrm{f}}\right) / 2\right)-B T_{\mathrm{C}}$.

The magnetic entropy change $\left(\Delta S_{\mathrm{M}}\right)$ can be obtained through the adiabatic change of temperature by the application of a magnetic field. $\Delta S_{\mathrm{M}}$ as a function of temperature for a field variation from 0 to $H_{\max }$ is given by

$$
\begin{aligned}
\Delta & S_{\mathrm{M}}\left(T, \Delta H=H_{\max }\right) \\
& =H_{\max }\left[-A\left(\frac{M_{\mathrm{i}}-M_{\mathrm{f}}}{2}\right) \operatorname{sech}^{2}\left(A\left(T_{\mathrm{C}}-T\right)\right)+B\right] .
\end{aligned}
$$

A more abrupt variation of magnetization near the magnetic transition occurs and results in a large magnetic entropy change. $\Delta S_{\mathrm{M}}$ depends on the temperature gradient of the magnetization and reaches a maximum value around $T_{\mathrm{C}}$.

Relative cooling power ( $\mathrm{RCP}$ ) is a useful parameter which decides the efficiency of magnetocaloric materials based on the magnetic entropy change [1,39]. The RCP is defined as the product of the maximum magnetic entropy change $\Delta S_{\mathrm{M} \max }$ and full-width at half-maximum in $\Delta S_{\mathrm{M}}(T)$ curve $\left(\delta T_{\mathrm{FWHM}}\right)$. According to this model, $\Delta S_{\mathrm{M} \max }$ is available by

$$
\Delta S_{\mathrm{M} \max }=H_{\max }\left[-A\left(\frac{M_{\mathrm{i}}-M_{\mathrm{f}}}{2}\right)+B\right]
$$

and $\delta T_{\mathrm{FWHM}}$ is presented by

$$
\delta T_{\mathrm{FWHM}}=\frac{2}{A} \cosh ^{-1}\left(\frac{2 A\left(M_{\mathrm{i}}-M_{\mathrm{f}}\right)}{A\left(M_{\mathrm{i}}-M_{\mathrm{f}}\right)+2 B}\right)^{1 / 2} .
$$

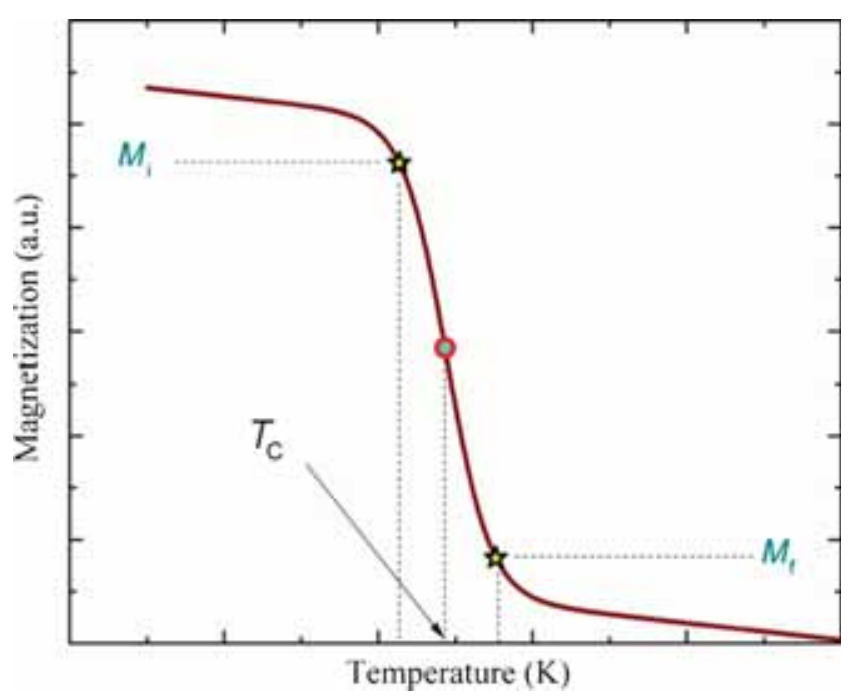

Figure 1. Temperature dependence of magnetization in constant applied magnetic field. 
Table 1. Model parameters for $\mathrm{La}_{0.67} \mathrm{Ca}_{0.33} \mathrm{Mn}_{0.9} \mathrm{Fe}_{0.1} \mathrm{O}_{3}$ at several magnetic fields.

\begin{tabular}{lccccc}
\hline$\mu_{0} H(T)$ & $M_{\mathrm{i}}\left(\mathrm{emu} \mathrm{g}^{-1}\right)$ & $M_{\mathrm{f}}\left(\mathrm{emu} \mathrm{g}^{-1}\right)$ & $T_{\mathrm{C}}(\mathrm{K})$ & $B\left(\mathrm{emu} \mathrm{g}^{-1} \mathrm{k}^{-1}\right)$ & $S_{\mathrm{C}}\left(\mathrm{emu} \mathrm{g}^{-1} \mathrm{k}^{-1}\right)$ \\
\hline 0.05 & 16.97393 & 0.45549 & 105.043 & -0.00289 & -0.41592 \\
0.1 & 25.47044 & 0.56936 & 106.19 & -0.00541 & -0.51 \\
0.2 & 33.14763 & 0.56936 & 105.665 & -0.00401 & -0.49491 \\
0.5 & 38.14338 & 2.22846 & 106.912 & -0.00842 & -0.49508 \\
1 & 41.25447 & 5.65958 & 109.444 & -0.0261 & -0.45866 \\
1.5 & 42.33495 & 9.47224 & 110.67 & -0.0475 & -0.43608 \\
2 & 45.28089 & 9.01723 & 112.563 & -0.04024 & -0.41307 \\
3 & 46.31226 & 14.34203 & 113 & -0.06545 & -0.39333 \\
\hline
\end{tabular}

Then RCP is computed by

$$
\begin{aligned}
\mathrm{RCP}=- & \Delta S_{\mathrm{M} \max } \delta T_{\mathrm{FWHM}}=H_{\max }\left(M_{\mathrm{i}}-M_{\mathrm{f}}-\frac{2 B}{A}\right) \\
& \times \cosh ^{-1}\left(\frac{2 A\left(M_{\mathrm{i}}-M_{\mathrm{f}}\right)}{A\left(M_{\mathrm{i}}-M_{\mathrm{f}}\right)+2 B}\right)^{1 / 2} .
\end{aligned}
$$

The RCP corresponds to the amount of heat that can be transferred between the cold and hot parts of the refrigerator in one ideal thermodynamic cycle. This parameter allows an easy comparison of different magnetic materials for applications in magnetic refrigeration; hence, larger RCP values lead to better magnetocaloric materials.

Another figure of merit which is used to compare the magnetic refrigerant materials is the refrigerant capacity (RC). The RC can be determinate by numerically integrating the area under the $\Delta S_{\mathrm{M}}(T)$ curve using the temperatures at half-maximum of the peak as the integration limits [40]. Here, $\mathrm{RC}$ value can be obtained as

$$
\begin{gathered}
\mathrm{RC}=-\int_{T_{\mathrm{C}}+\frac{\delta T_{\mathrm{FWHM}}}{2}}^{T_{\mathrm{C}}-\frac{\delta T_{\mathrm{FWHM}}}{2}}(T) \mathrm{d} T \\
=H_{\max }\left[-\left(M_{\mathrm{i}}-M_{\mathrm{f}}\right) \tanh \left(A \frac{\delta T_{\mathrm{FWHM}}}{2}\right)\right. \\
\left.+B \delta T_{\mathrm{FWHM}}\right] .
\end{gathered}
$$

The heat capacity can be calculated from the magnetic contribution to the entropy change induced in the material, $\Delta C_{\mathrm{P}, \mathrm{H}}$, by the following expression:

$$
\Delta C_{\mathrm{P}, \mathrm{H}}=T \frac{\partial \Delta S_{\mathrm{M}}}{\partial T} .
$$

From this model, a determination of $\Delta C_{\mathrm{P}, \mathrm{H}}$ can be carried out as follows:

$$
\begin{aligned}
\Delta C_{\mathrm{P}, \mathrm{H}}(T, \Delta H & \left.=H_{\mathrm{max}}\right) \\
=H_{\max } & {\left[-T A^{2}\left(M_{\mathrm{i}}-M_{\mathrm{f}}\right) \operatorname{sech}^{2}\left(A\left(T_{\mathrm{C}}-T\right)\right)\right.} \\
& \left.\times \tanh \left(A\left(T_{\mathrm{C}}-T\right)\right)\right] .
\end{aligned}
$$

From this phenomenological model, it can easily assess the values of $\Delta T_{\mathrm{FWHM}}, \Delta S_{\mathrm{M} \text { max }}, \mathrm{RCP} \mathrm{RC}$ and $\Delta C_{\mathrm{P}, \mathrm{H} \min / \max }$ for $\mathrm{La}_{0.67} \mathrm{Ca}_{0.33} \mathrm{Mn}_{0.9} \mathrm{Fe}_{0.1} \mathrm{O}_{3}$ at several magnetic fields.

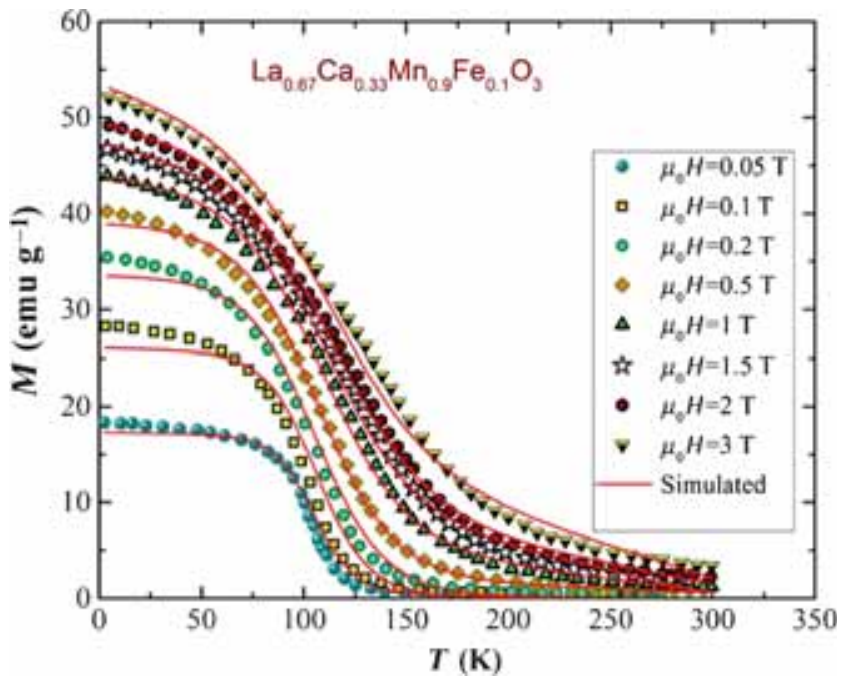

Figure 2. Magnetization vs. temperature for the $\mathrm{La}_{0.67} \mathrm{Ca}_{0.33}$ $\mathrm{Mn}_{0.9} \mathrm{Fe}_{0.1} \mathrm{O}_{3}$ system at several magnetic fields. The solid lines are modelled results and symbols represent experimental data from reference [34].

\section{Model application and discussions}

In order to predict the thermomagnetic properties for $\mathrm{La}_{0.67} \mathrm{Ca}_{0.33} \mathrm{Mn}_{0.9} \mathrm{Fe}_{0.1} \mathrm{O}_{3}$, numerical calculations were made with parameters as displayed in table 1. Figure 2 depicts the magnetization $v s$. temperature in different applied magnetic field shifts for $\mathrm{La}_{0.67} \mathrm{Ca}_{0.33} \mathrm{Mn}_{0.9} \mathrm{Fe}_{0.1} \mathrm{O}_{3}$ prepared by the solid-state reaction method as described elsewhere [34]. The symbols represent experimental data from reference [34], while the solid curves represent modelled data using model parameters given in table 1 . It is seen that for the given parameters, the results of calculation are in good accordance with the experimental results. The substitution of the manganese with the iron in the parent compound $\mathrm{La}_{0.67} \mathrm{Ca}_{0.33} \mathrm{MnO}_{3}$ leads to introduce the quenched disorder and has modified the nature of the phase transition from the first-order to the second-order-like [34]. Consequently magnetic transition in $\mathrm{La}_{0.67} \mathrm{Ca}_{0.33} \mathrm{Mn}_{0.9} \mathrm{Fe}_{0.1} \mathrm{O}_{3}$ is reversible in a cycle of increasing and decreasing temperature and the thermal hysteresis losses have been reduced which is highly desired in the sense of practical application. It can be 
reported that the magnetization exhibits a continuous change around $T_{\mathrm{C}}$ in different magnetic fields [34].

Furthermore, figures 3 and 4 show predicted values for changes of magnetic entropy and specific heat. Magnetic entropy change in $\mathrm{La}_{0.67} \mathrm{Ca}_{0.33} \mathrm{Mn}_{0.9} \mathrm{Fe}_{0.1} \mathrm{O}_{3}$ is reported for $\mu_{0} H=0.05,0.1,0.2,0.5,1,1.5,2$ and $3 \mathrm{~T}$ in figure 3 and shows an increase in $-\Delta S_{\mathrm{M}}$ with increasing $\mu_{0} H$. The $-\Delta S_{\mathrm{M}}$ is found to be positive in the entire temperature range for all magnetic fields confirmed the ferromagnetic character. It is seen that the results of calculation are in a good agreement with the experimental results. The MCE increases with the increase of the applied magnetic field and with the change of magnetization during application of magnetic field. This means that the effect reaches its maximum in the vicinity of magnetic phase transition points. The large values of $-\Delta S_{\mathrm{M}}$ for $\mathrm{La}_{0.67} \mathrm{Ca}_{0.33} \mathrm{Mn}_{0.9} \mathrm{Fe}_{0.1} \mathrm{O}_{3}$ compounds originate from a reversible second-order magnetic transition [34]. The magnetic transition becomes broader and so gives rise to a larger broadening of the $-\Delta S_{\mathrm{M}}$ [41]. The broad magnetic entropy peaks are advantage for magnetic cooling system [42].

Using equation (8) one can calculate the specific heat changes, $\Delta C_{\mathrm{P}, \mathrm{H}}$ caused by the applied magnetic field. Shown in figure 4 is $\Delta C_{\mathrm{P}, \mathrm{H}}$ as a function of temperature for $\mu_{0} H=$ $0.05,0.1,0.2,0.5,1,1.5,2$ and $3 \mathrm{~T}$. It is clearly seen that $\Delta C_{\mathrm{P}, \mathrm{H}}$ changes sharply from the negative to the positive at the Curie temperature. Since $\partial M / \partial T<0 ; \Delta S_{\mathrm{M}}<0$ results, and accordingly the total entropy decreases upon magnetization. Furthermore, $\Delta C_{\mathrm{P}, \mathrm{H}}<0$ for $T<T_{\mathrm{C}}$ and $\Delta C_{\mathrm{P}, \mathrm{H}}>0$ for $T>T_{\mathrm{C}}[43,44]$. The sum of the two parts is the magnetic contribution to the total specific heat which affects the cooling or heating power of the magnetic refrigerator [45]. Specific heat presents the advantage of delivering values necessary for further refrigerator design, should the material in question be selected.

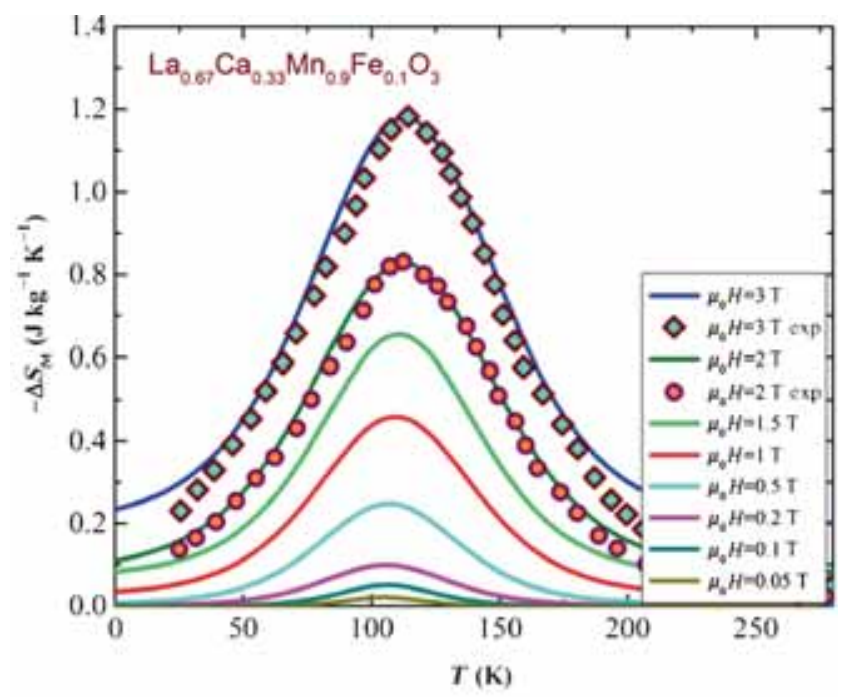

Figure 3. Magnetic entropy change, $\Delta S_{\mathrm{M}}$, for the $\mathrm{La}_{0.67} \mathrm{Ca}_{0.33} \mathrm{Mn}_{0.9} \mathrm{Fe}_{0.1} \mathrm{O}_{3}$ sample. The solid lines are predicted results and symbols represent experimental data from reference [34].
The MCE data of different materials of same universality class should fall onto the same curve irrespective of the applied magnetic field [46]. Because of the intrinsic relation between the MCE and the universality class, one can obtain the critical exponents based on the MCE data, which may be another method for determining the critical behaviour of phase transition, i.e., the universality class. Using the magnetocaloric response (or magnetic entropy change), one can investigate the nature of these coupled order parameters and further understand the rich magnetic properties exhibited by $\mathrm{La}_{0.67} \mathrm{Ca}_{0.33} \mathrm{Mn}_{0.9} \mathrm{Fe}_{0.1} \mathrm{O}_{3}$ manganites. At the peak, the field dependence of $-\Delta S_{\mathrm{M}}$ can be assumed to be a power law, with an exponent $n$

$$
\Delta S_{\max } \approx a\left(\mu_{0} H\right)^{n} .
$$

At $T_{\mathrm{C}}$, the exponent $n$ becomes field independent and is expressed as

$$
n\left(T_{\mathrm{C}}\right)=\left(\frac{\beta-1}{\beta+\gamma}\right)+1,
$$

where $\beta$ and $\gamma$ are the critical exponents [7,47]. With the Widom relation $\delta=1+\gamma / \beta$ [48], relation (10) can be written as

$$
n\left(T_{\mathrm{C}}\right)=\frac{1}{\delta}\left(1-\frac{1}{\beta}\right)+1 .
$$

As shown in figure 5 the fitting of full square points $\left(\Delta S_{\max }\right)$ leads to that $n\left(T_{\mathrm{C}}\right)=0.710(2)$ for $\mathrm{La}_{0.67} \mathrm{Ca}_{0.33} \mathrm{Mn}_{0.9} \mathrm{Fe}_{0.1} \mathrm{O}_{3}$, which is larger than the predicted value of 2/3 in the mean field approach [49] due to the local inhomogeneities in $\mathrm{La}_{0.67} \mathrm{Ca}_{0.33} \mathrm{Mn}_{0.9} \mathrm{Fe}_{0.1} \mathrm{O}_{3}$.

As discussed above, the width of $\Delta S_{\mathrm{M}}$ becomes more and more broad with the increase of field. In order to confirm this result; the field dependence of the full-width at halfmaximum $\Delta T_{\mathrm{FWHM}}$ is given in figure 5 . It is found that

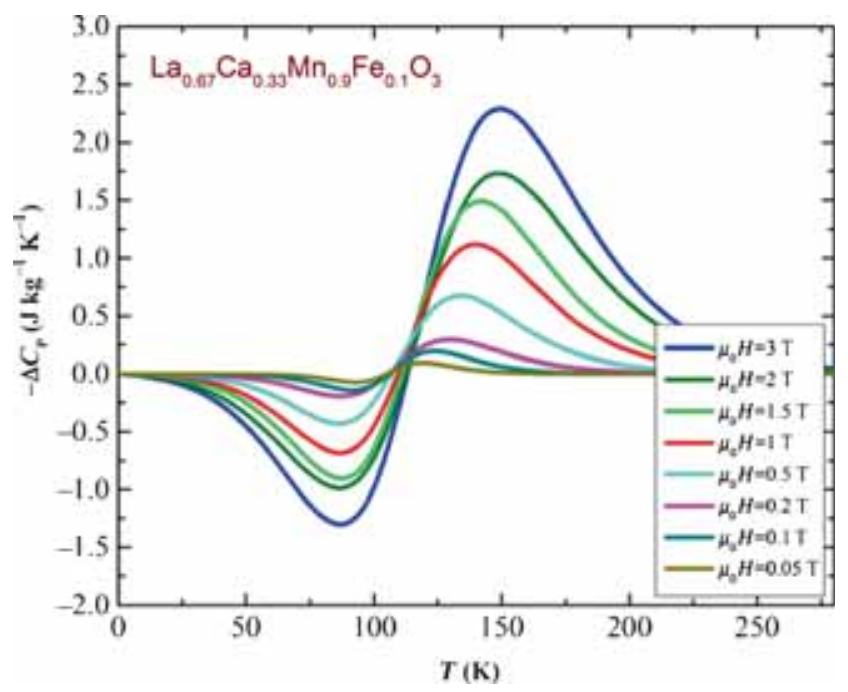

Figure 4. Heat capacity change, $\Delta C_{\mathrm{p}}$, for $\mathrm{La}_{0.67} \mathrm{Ca}_{0.33} \mathrm{Mn}_{0.9}$ $\mathrm{Fe}_{0.1} \mathrm{O}_{3}$ system at several magnetic fields. 


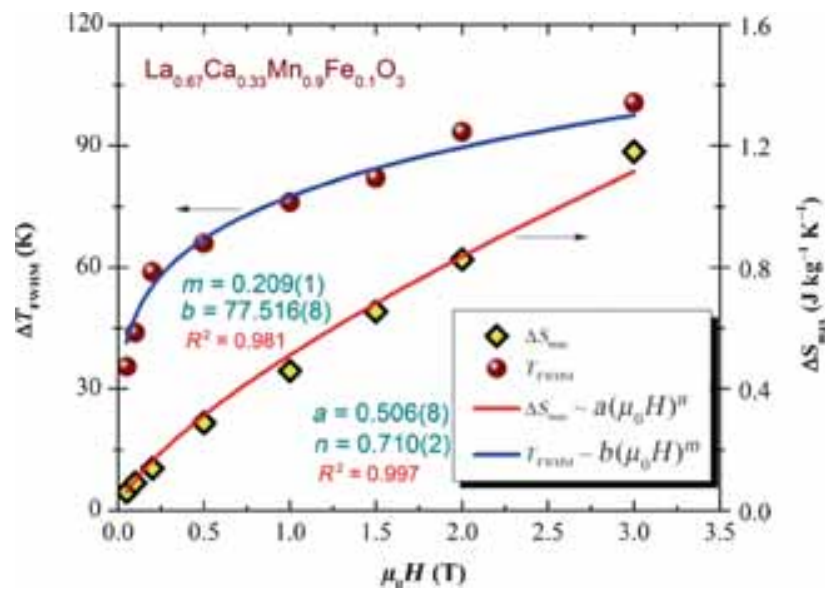

Figure 5. Field dependence of $\Delta S_{\max }$ and $\Delta T_{\text {FWHAM }}$ for $\mathrm{La}_{0.67} \mathrm{Ca}_{0.33} \mathrm{Mn}_{0.9} \mathrm{Fe}_{0.1} \mathrm{O}_{3}$.

$\Delta T_{\mathrm{FWHM}}$ is also field dependent. The fitness of $\Delta T_{\mathrm{FWHM}}$ gives that $\Delta T_{\mathrm{FWHM}} \approx b\left(\mu_{0} H\right)^{m}$ where $b=77.516(8)$ and $m=0.209(1)$.

In addition to the magnitude of the $\Delta S_{\mathrm{M}}$, other important parameters used to characterize the refrigerant efficiency of the material are the RCP and the RC defined as equations (5) and (6). RCP and RC give an estimate of quantity of the heat transfer between the hot $\left(T_{\text {hot }}\right)$ and cold $\left(T_{\text {cold }}\right)$ end during one refrigeration cycle and is the area under the $\Delta S_{\mathrm{M}}$ vs. $T$ curve between two temperatures $\left(\Delta T=T_{\text {hot }}-T_{\text {cold }}\right)$ of the full-width at half-maximum (FWHM) of the curve. Figure 6 shows the values for the compound $\mathrm{La}_{0.67} \mathrm{Ca}_{0.33} \mathrm{Mn}_{0.9} \mathrm{Fe}_{0.1} \mathrm{O}_{3}$ as a function of applied magnetic field.

To analyse the nature of the magnetic phase transition in detail, a critical exponent's study near their Curie temperature $\left(T_{\mathrm{C}}\right)$ for the $\mathrm{La}_{0.67} \mathrm{Ca}_{0.33} \mathrm{Mn}_{0.9} \mathrm{Fe}_{0.1} \mathrm{O}_{3}$ sample was carried out. In this context, the field dependence of $\Delta S_{\mathrm{M}}$ is used for investigating the critical behaviour [50,51]. On the basis of figure 6, the field dependence of the RCP for $\mathrm{La}_{0.67} \mathrm{Ca}_{0.33} \mathrm{Mn}_{0.9} \mathrm{Fe}_{0.1} \mathrm{O}_{3}$ is depicted. It can be expressed as a power law by taking account of the field dependence of entropy change $\Delta S_{\mathrm{M}}$ and reference temperature into consideration $\mathrm{RCP}=v\left(\mu_{0} H\right)^{w}$ (where $w=1+1 / \delta, v$ is a constant and $\delta$ the critical exponent of the magnetic transition), as predicted by scaling laws [35]. This feature provides insight into the critical behaviour of the compound. The lowest field data have been omitted because of the large uncertainty of the peak position. The slope of the linear function fitted to the Ln-Ln data yields the value of $w \approx 1.208(7)$ which implies $\delta=4.791(5)$.

The knowledge of two critical exponents $n$ and $\delta$ inferred from the field dependence of MCE is enough to determine other exponents, $\beta$ and $\gamma$. By means of the Widom relation, equations (10) and (11) one effortlessly finds $\beta=1 /(\delta(1-n)$ $+1) \approx 0.418(6)$, and $\gamma=\beta(\delta-1) \approx 1.587(3)$. Fundamentally $\beta$ and $\gamma$ keep close to the value expected for the 3D Heisenberg ferromagnets with ferromagnetic shortrange interactions. A similar result of critical behaviours was

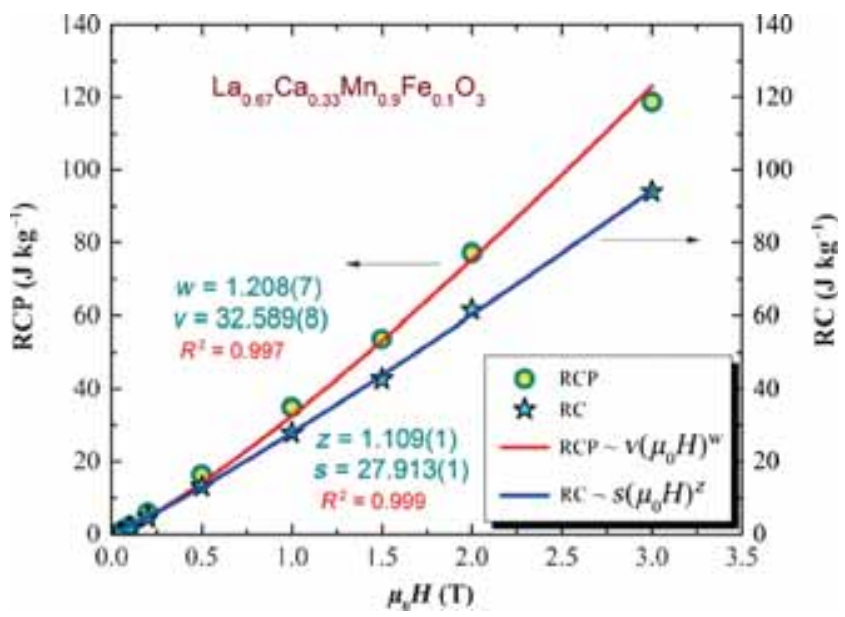

Figure 6. Field dependence of RCP and RC for $\mathrm{La}_{0.67} \mathrm{Ca}_{0.33} \mathrm{Mn}_{0.9} \mathrm{Fe}_{0.1} \mathrm{O}_{3}$.

observed in Fe-doped $\mathrm{LaMnO}_{3}$ [52] and $\mathrm{ErTiO}_{3}$ [46]. The obtained critical exponents of $\mathrm{La}_{0.67} \mathrm{Ca}_{0.33} \mathrm{Mn}_{0.9} \mathrm{Fe}_{0.1} \mathrm{O}_{3}$, as well as those of different theoretical models, are listed in table 2 for comparison.

Hence, the present study deals with critical and magnetocaloric properties of $\mathrm{La}_{0.67} \mathrm{Ca}_{0.33} \mathrm{Mn}_{0.9} \mathrm{Fe}_{0.1} \mathrm{O}_{3}$. A precise estimate of the critical exponents for this material near ferromagnetic (FM)-paramagnetic (PM) phase transition temperature is presented and shows that the critical exponents are 3D short-range Heisenberg like in this particular compound. This reflects the existence of FM short-range order in $\mathrm{La}_{0.67} \mathrm{Ca}_{0.33} \mathrm{Mn}_{0.9} \mathrm{Fe}_{0.1} \mathrm{O}_{3}$. The mixed valence of $\mathrm{Mn}$ and Fe ions promotes both the FM double-exchange and AFM super-exchange interactions and thus leads to inhomogeneous regions in magnetism.

In recent times, the authors [22,26,53] and others [54,55] have shown that for second-order magnetic transition materials the $\Delta S_{\mathrm{M}}(T)$ curves obtained with different maximum applied fields will collapse onto a universal curve by normalizing all the $\Delta S_{\mathrm{M}}$ curves with their peak entropy change, respectively, as $\Delta S_{\mathrm{M}^{\prime}}=\Delta S_{\mathrm{M}} / 2$, and the temperature axis has to be rescaled in a different way below and above $T_{\mathrm{C}}$, just by imposing that the position of two additional reference points in the curve correspond to new parameter $\theta$, defined by the expression $[26,56]$

$$
\theta=\left\{\begin{array}{c}
-\left(T-T_{\mathrm{C}}\right) /\left(T_{\mathrm{r} 1}-T_{\mathrm{C}}\right) ; \quad T \leq T_{\mathrm{C}}, \\
\left(T-T_{\mathrm{C}}\right) /\left(T_{\mathrm{r} 2}-T_{\mathrm{C}}\right) ; \quad T>T_{\mathrm{C}},
\end{array}\right.
$$

where $T_{\mathrm{r} 1}$ and $T_{\mathrm{r} 2}$ are the reference temperatures below and above $T_{\mathrm{C}}$, respectively.

The phenomenological construction of the universal scaling of different magnetic field is depicted in figure 7. Figure 7 shows an attempt to form a master curve for the entropy change of the $\mathrm{La}_{0.67} \mathrm{Ca}_{0.33} \mathrm{Mn}_{0.9} \mathrm{Fe}_{0.1} \mathrm{O}_{3}$. One can see that the universal behaviour manifests itself only in a limited interval around the peak temperature. Farther below and above the peak the scaling behaviour apparently breaks. Increasing fields produce an increase in the reduced magnetic 
Table 2. Critical exponents of materials obtained from the MCE scaling law method and theoretical values of three models.

\begin{tabular}{|c|c|c|c|c|c|}
\hline Composition & $n$ & $\beta$ & $\gamma$ & $\delta$ & Ref. \\
\hline $\mathrm{La}_{0.67} \mathrm{Ca}_{0.33} \mathrm{Mn}_{0.9} \mathrm{Fe}_{0.1} \mathrm{O}_{3}$ & $0.710(2)$ & $0.418(6)$ & $1.587(3)$ & $4.791(3)$ & Present \\
\hline $\mathrm{DyTiO}_{3}$ & $0.621(4)$ & $0.352(2)$ & $1.355(3)$ & $4.85(2)$ & [46] \\
\hline $\mathrm{HoTiO}_{3}$ & $0.679(3)$ & $0.397(5)$ & $1.476(4)$ & $4.72(6)$ & {$[46]$} \\
\hline $\mathrm{ErTiO}_{3}$ & $0.710(7)$ & $0.413(5)$ & $1.611(6)$ & $4.90(5)$ & {$[46]$} \\
\hline $\mathrm{La}_{0.6} \operatorname{Pr}_{0.4} \mathrm{Fe}_{10.7} \mathrm{Co}_{0.8} \mathrm{Si}_{1.5}$ & $0.773(5)$ & $0.560(5)$ & $1.381(1)$ & $3.463(9)$ & [36] \\
\hline Tricritical mean-field model & - & 0.25 & 1.0 & 5.0 & {$[61]$} \\
\hline Mean-field model & - & 0.5 & 1.0 & 3.0 & {$[61]$} \\
\hline 3D Heisenberg model & - & 0.365 & 1.386 & 4.8 & {$[61]$} \\
\hline 3D Ising model & - & 0.325 & 1.24 & 4.82 & [61] \\
\hline
\end{tabular}

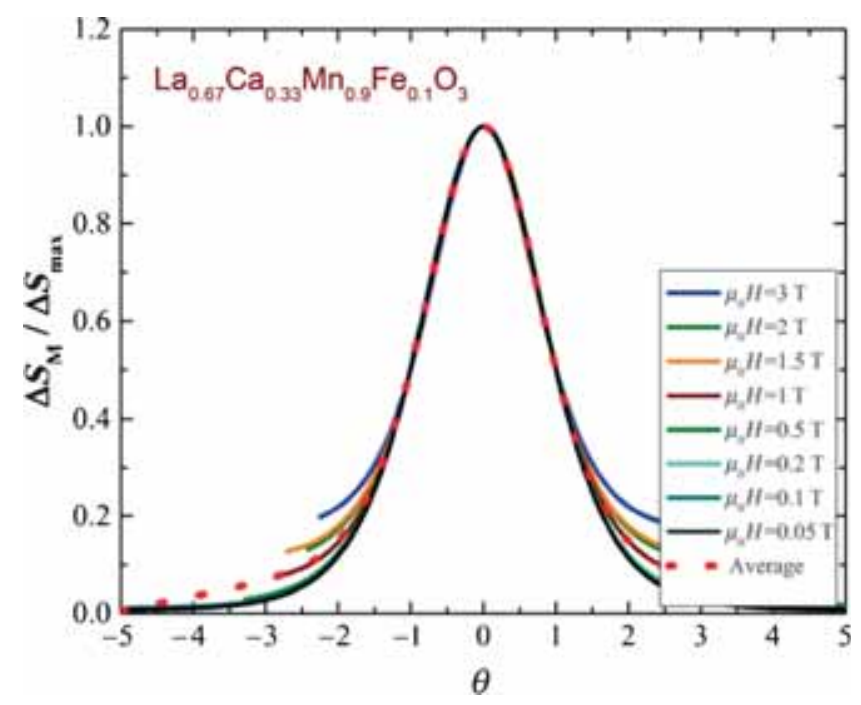

Figure 7. Normalized entropy change as a function of the rescaled temperature $\theta$ (the dash curve is averaged).

entropy change. Deviations from the collapse might indicate either the influence of the demagnetizing field associated to the shape of the sample [57] or the presence of additional magnetic phases $[58,59]$. The divergence of the curves is clear in the compound, particularly above the $T_{\mathrm{C}}$. As you can see, this universal curve is independent of the external measurement conditions, which is just determined by the intrinsic magnetization.

On the basis of the universal scaling, an average curve is obtained, as shown as dashed curve in figure 7 which gives a smoother description of the curve. This average curve, once the temperature axis is back transformed from the reduced temperature to the unnormalized one, allows making extrapolations to lower temperatures for the high field data and obtaining a finer description of the peak for the low field curves [60].

\section{Conclusions}

In summary, the exhibition of large MCE in $\mathrm{La}_{0.67} \mathrm{Ca}_{0.33}$ $\mathrm{Mn}_{0.9} \mathrm{Fe}_{0.1} \mathrm{O}_{3}$ which is associated with a FM to PM phase transition near the Curie temperature was reported.
Dependence of the magnetization on temperature variation for $\mathrm{La}_{0.67} \mathrm{Ca}_{0.33} \mathrm{Mn}_{0.9} \mathrm{Fe}_{0.1} \mathrm{O}_{3}$ upon different magnetic fields was simulated. A good RCP value was observed. The large change of magnetization during the phase transition leads to large MCE. It was shown that the higher field $\mathrm{La}_{0.67} \mathrm{Ca}_{0.33} \mathrm{Mn}_{0.9} \mathrm{Fe}_{0.1} \mathrm{O}_{3} \mathrm{MCE}$ has a power law field dependence characterized by an exponent $n=0.710(2)$. The critical exponents associated with FM transition have been determined from the MCE methods. Therefore, the MCE scaling laws can be applied to complex magnetic systems involving different magnetization processes in the critical region. Furthermore, the $\Delta S_{\mathrm{M}}$ collapse onto a universal curve based on the scaling relation, where an average curve was obtained. This sample exhibits considerably no magnetic hysteresis, which was beneficial for the magnetic cooling efficiency. In this respect, the lanthanum manganites based materials are very promising refrigerants because of (1) high safety, (2) easily tuned Curie temperature, (3) relatively low cost of components and fabrication, and (4) being non-toxic.

\section{Acknowledgement}

This study was supported by the Tunisian Ministry of Higher Education and Scientific Research.

\section{References}

[1] Tishin A M and Spichkin Y I 2003 The Magnetocaloric Effect and Its Applications (Bristol: Institute of Physics Publishing)

[2] Pecharsky V K and Gschneidner K A Jr 1997 Phys. Rev. Lett. 784494

[3] Pecharsky V K and Gschneidner K A Jr 2001 Adv. Mater. 13 683

[4] M'nassri R and Cheikhrouhou A 2014 J. Supercond. Nov. Magn. 271059

[5] Mbarek H, M'nasri R, Cheikhrouhou-Koubaa W and Cheikhrouhou A 2014 Status Solidi A 211975

[6] Fähler S, Rössler U K, Kastner O, Eckert J, Eggeler G, Emmerich H, Entel P, Müller S, Quandt E and Albe K 2012 Adv. Eng. Mater. 1410

[7] De Oliveira N A and Von Ranke P J 2010 Phys. Rep. 48989

[8] Phan M H and Yu S C 2007 J. Magn. Magn. Mater. 308325 
[9] M'nassri R, Chniba Boudjada N and Cheikhrouhou A 2015 J. Alloys Compd. 62620

[10] M'nassri R, Cheikhrouhou-Koubaa W, Chniba-Boudjada N and Cheikhrouhou A 2013 J. Appl. Phys. 113073905

[11] Choura Maatar S, M'nassri R, Cheikhrouhou Koubaa W, Koubaa M and Cheikhrouhou A 2015 J. Solid State Chem. 22583

[12] M'nassri R, Chniba Boudjada N and Cheikhrouhou A $2015 \mathrm{~J}$. Alloys Compd. $\mathbf{6 4 0} 183$

[13] M'nassri R and Cheikhrouhou A 2014 J. Supercond. Nov. Magn. 27421

[14] Akther Hossain A K M, Cohen L F, Kodenkandeth T, Mac Manus-Driscoll J and Mc Nalford N 1999 J. Magn. Magn. Mater. 19531

[15] Selmi A, Bettaibi A, Rahmouni H, M'nassri R, Chniba Boudjada N, Chiekhrouhou A and Khirouni K 2015 J. Ceram. Int. $\mathbf{4 1} 11221$

[16] M'nassri R and Cheikhrouhou A 2014 J. Supercond. Nov. Magn. 271463

[17] Zener C 1951 Phys. Rev. 82403

[18] Millis A J, Littlewood P B and Shraiman B I 1995 Phys. Rev. Lett. 745144

[19] Goodenough J B, Wold A, Arnott R J and Menyuk N 1961 Phys. Rev. 124373

[20] M'nassri R, Cheikhrouhou-Koubaa W, Boudjada N and Cheikhrouhou A 2013 J. Supercond. Nov. Magn. 261429

[21] M'nassri R, Cheikhrouhou-Koubaa W, Koubaa M, Boudjada $\mathrm{N}$ and Cheikhrouhou A 2011 Solid State Comm. 1511579

[22] M'nassri R, Cheikhrouhou-Koubaa W, Kouba M and Cheikhrouhou A 2012 IOP Conf. Ser: Mater. Sci. Eng. 28 012050

[23] Klein J, Höfener C, Uhlenbruck S, Alff L, Büchner B and Gross R 1999 Europhys. Lett. 47371

[24] M'nassri R, Chniba Boudjada N and Cheikhrouhou A $2015 \mathrm{~J}$. Alloys Compd. $\mathbf{6 2 6} 20$

[25] Lampen P, Puri A, Phan M-H and Srikanth H 2012 J. Alloys Compd. $\mathbf{5 1 2} 94$

[26] M'nassri R and Cheikhrouhou A 2014 J. Supercond. Nov. Magn. 271463

[27] Phan M H and Yu S C 2007 J. Magn. Magn. Mater. 308325

[28] Osthöver C, Grünberg P and Arons R R 1998 J. Magn. Magn. Mater. 854177

[29] Selmi A, M'nassri R, Cheikhrouhou-Koubaa W, Chniba Boudjada N and Cheikhrouhou A 2015 J. Alloys Compd. 619 627

[30] Barahona P, Pena O, Antunes A B, Campos C, Pecchi G, Moreno Y, Moure C and Gil V 2008 J. Magn. Magn. Mater. 320 e61

[31] Selmi A, M'nassri R, Cheikhrouhou-Koubaa W, Chniba Boudjada N and Cheikhrouhou A 2015 J. Ceram. Int. 417723

[32] Mahjoub S, Baazaoui M, M'nassri R, Rahmouni H, Chniba Boudjada N and Oumezzine M 2014 J. Alloys Compd. 608 191

[33] Chang Y L, Huang Q and Ong C K 2002 J. Appl. Phys. 91789
[34] Mukadam M D and Yusuf S M 2009 J. Appl. Phys. 105 063910

[35] Oumezzine M, Pea O, Kallel S and Oumezzine M $2012 \mathrm{~J}$. Alloys Compd. $\mathbf{5 3 9} 116$

[36] M'nassri R 2014 J. Supercond. Nov. Magn. 271787

[37] Halder M, Yusuf S M, Mukadam M D and Shashikala K 2010 Phys. Rev. B 81174402

[38] Hamad M A 2012 Phase Transitions 85106

[39] Gschneidner K A and Pecharsky V K Jr 2000 Annu. Rev. Mater. Sci. 30387

[40] Gschneidner K A, Pecharsky V K, Pecharsky A O and Zimm C B 1999 Mater. Sci. Forum 31569

[41] El-Hagary M 2010 J. Alloys Compd. 502376

[42] Wang A, Liu Y, Zhang Z, Long Y and Cao G 2004 Solid State Commun. 130293

[43] Foldeaki M, Chahine R and Bose T K 1995 J. Appl. Phys. 77 3528

[44] Yang H, Zhu Y H, Xian T and Jiang J L 2013 J. Alloys Compd. 555150

[45] Zhang X X, Wen G H, Wang F W, Wang W H, Yu C H et al 2000 Appl. Phys. Lett. 773072

[46] Su Y, Sui Y, Cheng J-G, Zhou J-S, Wang X, Wang Y and Goodenough J B 2013 Phys. Rev. B 87195102

[47] Franco V and Conde A 2010 Int. J. Refrig. 33465

[48] Stanley H E 1971 Introduction to Phase Transitions and Critical Phenomena (London: Oxford University Press)

[49] Franco V, Blazquez J S and Conde A 2006 Appl. Phys. Lett. 89222512

[50] Franco V, Conde A, Romero-Enrique J M and Blazquez J S 2008 J. Phys. Condens. Matter 20285207

[51] Zhang X X, Tejada J, Xin Y, Sun G F, Wong K W and Bohigas X 1996 Appl. Phys. Lett. 693596

[52] Phan T-L, Thanh P Q, Yen P D H, Zhang P, Thanh T D and Yu S C 2013 Solid State Commun. 16749

[53] M'nassri R and Cheikhrouhou A 2014 J. Korean Phys. Soc. 64879

[54] Franco V, Conde A, Pecharsky V K and Gschneidner K A Jr 2007 Europhys. Lett. 7947009

[55] Franco V, Conde A, Sidhaye D, Prasad B L V, Poddar P, Srinath S, Phan M H and Srikanth H 2010 J. Appl. Phys. 107 09A902

[56] Franco V, Blazquez J S and Conde A 2006 Appl. Phys. Lett. 89222512

[57] Caballero-Flores R, Franco V, Conde A and Kiss L F $2009 \mathrm{~J}$. Appl. Phys. 105 07A919

[58] Franco V, Conde A, Provenzano V and Shull R D $2010 \mathrm{~J}$. Magn. Magn. Mater. 322218

[59] Franco V, Caballero-Flores R, Conde A, Dong Q Y and Zhang H W 2009 J. Magn. Magn. Mater. 3211115

[60] Zhang L, Fan J, Tong W, Ling L, Pi L and Zhang Y 2012 Physica B 4073543

[61] Stanley H E 1999 Rev. Mod. Phys. 71 S358 